\title{
inut \\ Can Heritage Speakers Predict Lexical and Morphosyntactic Information in Reading?
}

\author{
Olga Parshina ${ }^{1, *}$, Anastasiya Lopukhina ${ }^{1,2}$ and Irina A. Sekerina ${ }^{1,3}$ (1) \\ 1 Center for Language and Brain, HSE University, 101000 Moscow, Russia; alopukhina@hse.ru (A.L.); \\ irina.sekerina@csi.cuny.edu (I.A.S.) \\ 2 Department of Theoretical Semantics, Vinogradov Russian Language Institute, 119019 Moscow, Russia \\ 3 Department of Psychology, College of Staten Island of the City University of New York, \\ New York, NY 10314, USA \\ * Correspondence: oparshina@hse.ru
}

check for updates

Citation: Parshina, Olga, Anastasiya Lopukhina, and Irina A. Sekerina. 2022. Can Heritage Speakers Predict Lexical and Morphosyntactic Information in Reading? Languages 7: 60. https://doi.org/10.3390/ languages7010060

Academic Editor: Ana I. Schwartz

Received: 16 October 2021

Accepted: 24 February 2022

Published: 4 March 2022

Publisher's Note: MDPI stays neutral with regard to jurisdictional claims in published maps and institutional affiliations.

Copyright: (C) 2022 by the authors. Licensee MDPI, Basel, Switzerland. This article is an open access article distributed under the terms and conditions of the Creative Commons Attribution (CC BY) license (https:// creativecommons.org/licenses/by/ $4.0 /)$.

\begin{abstract}
Ample evidence suggests that monolingual adults can successfully generate lexical and morphosyntactic predictions in reading and that correct predictions facilitate sentence comprehension. In this eye-tracking corpus reading study, we investigate whether the same is true for reading in heritage language. Specifically, we ask whether heritage speakers (HSs) of Russian are able to anticipate lexical and/or morphosyntactic information of the upcoming words in the sentence and whether they differ in the predictions from monolingual children and L2 learners. We are also interested in whether the literacy level (i.e., Russian literacy experience or reading fluency in English) influences lexical and morphosyntactic prediction. Our results indicate that HSs as well as other groups were able to anticipate the specific lexical item, and the ability was contingent on the Russian literacy experience and reading fluency in dominant English as evident in some of the early and late eye-tracking measures. Similar to children and L2 learners, the word class and the verb number predictability affected reading times in HSs, but HSs were the only group to anticipate the number of the upcoming noun. We discuss findings in respect to the utility account of the bilingual prediction and divergent attainment trajectory of the heritage language development.
\end{abstract}

Keywords: prediction; lexical prediction; morphosyntactic prediction; heritage speakers; Russian; reading; eye-tracking

\section{Introduction}

Research on language prediction in monolinguals has provided ample evidence that monolingual adults can predict the upcoming information in listening and reading (see Pickering and Gambi 2018, for review). Specifically, when monolingual adults read, they can successfully generate both lexical (i.e., activation of a specific lexical item and its grammatical form) and morphosyntactic predictions (i.e., activation of morphosyntactic features such as word class, noun gender, number, person and verb tense) from the context of the sentence. These predictions greatly facilitate language comprehension (Balota et al. 1985; Boston et al. 2008; Demberg and Keller 2008; Huettig 2015). Although the findings from the second language (L2) research are not as straightforward, there is an emerging consensus that L2 learners also can predict the upcoming linguistic information, but the extent of the prediction varies greatly dependent on multiple factors. One of the most important factors is the L2 exposure that includes the manner of L2 acquisition, i.e., when, where and how the L2 was learned (Kaan 2014; Kaan and Grüter 2021).

Surprisingly, very little is known about prediction abilities of heritage speakers (HSs), a bilingual group that differs from L2 learners in the timing, environment and mode of non-dominant language acquisition (Montrul 2008). Unlike typical L2 learners who study how to read and write in the L2 in schools or universities starting in late childhood or adolescence, HSs acquire heritage language at home from birth with most of the input being 
in the auditory modality. HSs then switch to the majority language at around the school age. With the onset of this switch, the amount of heritage language input and output typically plummets, resulting in the developmental 'freeze' of the heritage language abilities. In the literature, such developmental outcome is termed as 'divergent attainment' and it predicts that adult HSs would resemble young school-age monolingual children in language abilities (Polinsky and Scontras 2020), including reading skills (Parshina et al. 2021a, 2021b) and likely including prediction abilities in reading.

In this eye-tracking study, we extend an investigation of predictive processing in bilingual comprehension to HSs and address the question of whether the differences in the manner of the non-dominant (i.e., heritage) language acquisition between HSs and L2 learners and similarities between HSs and children influence their prediction abilities in heritage language comprehension. Specifically, we examined whether English-dominant HSs of Russian are able to predict the upcoming lexical and morphosyntactic information when reading isolated sentences in the heritage language and whether these predictive abilities differ from those of monolingual children and L2 learners of Russian.

We start with a brief overview of one of the most recent accounts of the prediction in bilingual language comprehension and discuss what this account implies for the prediction in the heritage language. Next, we review what is already known about how HSs predict reading in Russian. We conclude the introduction with specific research questions and hypotheses for this study.

\subsection{Prediction in L2 Bilingual Comprehension}

The research on predictive processing in L2 comprehension provides a full range of findings, from no evidence of the prediction in some studies (Grüter et al. 2012; Ito et al. 2017; Lew-Williams and Fernald 2010; Martin et al. 2013; Mitsugi and Macwhinney 2016) to native-like prediction in others (Foucart et al. 2014; Hopp 2013). In a recent review, Kaan and Grüter (2021), following Kuperberg and Jaeger (2016), suggest that the explanation for such variability lies in the utility of the prediction for L2 learners, encompassed by the question: is it worth engaging in the prediction considering all the factors that can aid it or, vice versa, make comprehension even more laborious? To answer this question, L2 learners, according to the utility account, adjust the predictive behavior after conducting the "cost-benefit analysis": they weigh the benefits of engaging in prediction (e.g., speeding up the comprehension) against its cost. If the task is too demanding, as in, for example, reading in a script that is different from their L1 (e.g., Cyrillic vs. Roman), and the L2 proficiency is very low, then the most efficient strategy is likely to not engage much in prediction (i.e., lexical or morphosyntactic) but 'focus' exclusively on the current information integration. On the other hand, for a balanced bilingual with lots of exposure to written materials in the L2, reading itself should not represent an issue and prediction is likely to be an efficient and not too costly way to facilitate language comprehension.

Indeed, in an eye-tracking study with 124 fairly proficient younger and older EnglishFrench and French-English bilinguals, Whitford and Titone (2017) reported that higher predictability of the words in the paragraph decreased reading times in all eye-movement measures under investigation (gaze duration, total reading time, skipping, and regression probabilities). The predictability effect stayed age-invariant (i.e., both younger and older adults were sensitive to the word predictability in reading) and language-invariant (i.e., the word predictability affected eye-movement measures both during L1 and L2 reading) (see also Libben and Titone 2009; Martin et al. 2013). Accordingly, in the few studies with unbalanced bilinguals or bilinguals reading in different scripts, the word predictability effects, while still present, were larger when reading in L2 compared with reading in L1 (Gollan et al. 2011 in overt naming) and were detected only in late eye-movement measures (e.g., Mor and Prior 2021).

Kaan and Grüter (2021) also suggest that another factor that L2 bilinguals weigh into the cost-benefit analysis is whether predictive cues from L1, especially morphosyntactic ones, can be transferred to L2. For example, gender- or case-marking cues for prediction in 
L2 can be used more efficiently when bilinguals' L1 is also a gender-marked language and has the case system (Dussias et al. 2013; Foucart et al. 2014, 2016; Frenck-Mestre et al. 2019; Hopp and Lemmerth 2018). Such prediction is less likely when the transfer of the cues from L1 is not possible (Dussias et al. 2013 for L2 English speakers; Foucart 2021 for review; Mitsugi and Macwhinney 2016) or they are not as salient as in L2 (Molinaro et al. 2017). With higher global proficiency in L2, including greater L2 exposure, however, bilinguals show more sensitivity to the L2-specific morphosyntactic features and are able to use them as stand-alone predictive cues in L2 comprehension (Dussias et al. 2013; Henry et al. 2020; Hopp 2013, 2015; cf. Mitsugi 2020). In addition, as L2 bilinguals reach higher levels of proficiency in L2, they begin to spend less time and resources on the lexical access and engage more in morphosyntactic planning which in turn results in a better ability to anticipate morphosyntactic information in a native-like manner (see Ito and Pickering 2021, for review).

\subsection{Prediction in Heritage Language Bilingual Comprehension}

Most of the research that directly or (more often) indirectly examines prediction in heritage language comprehension focuses on morphosyntax, specifically on the HSs' ability to anticipate the morphological features of gender, number or case. Since HSs often struggle with written language comprehension, the majority of the studies investigated the facilitatory effects of the grammatical cues in auditory comprehension. In general, the findings indicate that HSs are able to rely on the grammatical cues to anticipate the upcoming linguistic information in the auditory modality, especially in studies with highproficient HSs or with language pairs that have close typological proximity (in line with theoretical predictions of the utility account, Kaan and Grüter 2021).

In a recent visual world eye-tracking (VWP) study, Fuchs (2021) showed that HSs use gender information on the preceding article to speed up word recognition of the upcoming nouns in the same manner as the monolingual control group. Twenty-one English-dominant HSs of Spanish listened to the auditory stimuli containing the definite article with either masculine or feminine gender marker. At the same time, participants were presented with the visual images of two objects whose names either matched or mismatched the article in the grammatical gender. The results indicate that similar to monolinguals, HSs were faster in fixating the target object in the mismatch condition than in the match condition, although overall slower than monolinguals across two conditions.

Very similar results were obtained in the earlier set of the VWP studies, now with English-dominant HSs of Russian (Sekerina 2015, for review). Thirty-nine HSs (along with monolingual adults and children) viewed animated scenes while listening to the sentences that contained double cues on grammatical gender and number agreement (agreement markers on the adjective and the verb preceding the noun). As in Fuchs (2021), HSs of Russian again used gender markers to anticipate the upcoming noun, but they were slower than monolinguals and only used the feminine markers predictively. Notably, all three groups used the plural marker as the most consistent cue for prediction with no quantitative difference between monolinguals and HSs.

In general, based on the findings of the previous research, it appears that for HSs, the morphosyntactic cues that can be transferred from the dominant language (e.g., noun number or verbal tense for HSs of Russian with dominant English) or the cues that have consistent, transparent and perceptually salient markers (e.g., feminine markers on adjectives modifying nouns in Russian) have the strongest predictive power, at least in the spoken modality. This trend corresponds to what we typically observe in heritage language when analyzing the production errors or accuracy in auditory comprehension: the most difficulties occur in gender agreement with markers that are not perceptually salient or when the agreeing constituents are not adjacent. In auditory comprehension (and also in written comprehension in Gor 2019), verbal aspect and verbs of motion seem to present the most challenges (Gor 2019; Polinsky and Scontras 2020). Verbal tense, on the other hand, confirms to be the morphosyntactic phenomenon that is resilient to errors in many heritage 
languages (Polinsky and Scontras 2020, for review). These observations from production and auditory comprehension give us some insights as to which morphosyntactic cues might be used as predictive (i.e., noun number and verb tense) and which might not (i.e., noun gender and noun case) in written comprehension by HSs. Notably, they are remarkably in line with the predictions of the utility account as well (Kaan and Grüter 2021).

However, what do we know already about the heritage language prediction in the written modality? The studies that touch upon both full lexical prediction and morphosyntactic prediction in heritage language comprehension in the written modality are hard to find. For example, to test if HSs engage in the full lexical prediction (i.e., the prediction of the specific lexical item and its form, Luke and Christianson 2016), one should examine the HSs' performance on a cloze probability task (i.e., filling in the blanks while reading) (Taylor 1953) after it was normed in the HS population. Unfortunately, we are not aware of any studies with such norming procedures, as they are difficult to carry out from the practical standpoint (e.g., the majority of HSs cannot write). However, there are a few studies that used the cloze task, either non-normed or normed with the adult native speakers, as an approximate measure of HSs' proficiency level. These studies report higher performance accuracies of HSs on these tests compared with L2 learners (Luchkina et al. 2021) and greater predictive value of the cloze test for the assessment of the grammatical knowledge of HSs (Gor 2019). Thus, there is some initial evidence that literate HSs can generate predictions in heritage language written production. We are not aware of any studies, however, that take a step further and investigate prediction in heritage language comprehension online, specifically prediction in reading. In this eye-tracking corpus-based reading study, we aim to fill in the gap by examining both full lexical and morphosyntactic prediction in the written modality in heritage Russian.

\subsection{Prediction in Reading in Russian: What Do Eye Movements Tell Us?}

Recently, Lopukhina et al. (2021) directly investigated lexical and morphosyntactic prediction in monolingual reading in Russian. In this eye-tracking experiment, 96 adult native speakers of Russian read 144 sentences from the Russian Sentence Corpus (Laurinavichyute et al. 2019) that were previously normed in a cloze task by another 603 Russian adult speakers. The findings for the lexical prediction confirmed the predictability effect (Kliegl et al. 2004): in all dependent eye-movement measures (first fixation, single fixation, gaze duration and total reading time), the fixation duration on the current words decreased when the cloze probability of the lexical item increased.

Among morphosyntactic features, Lopukhina et al. (2021) investigated the prediction of the word class (both content and function words) as well as number, gender and case for nouns and tense, person, number and gender for verbs. As in the lexical prediction, the higher cloze probabilities of the word class decreased reading times on the current word, suggesting that readers anticipated part-of-speech of the upcoming words in the sentence. Higher morphological probabilities for the noun gender and the verb tense (future and present) decreased reading times of the currently fixated words (total reading time for noun gender and verb tense plus gaze duration for the verb tense). In general, the results of the study indicate that monolingual skilled readers of Russian pre-activate both lexical and morphosyntactic information in reading to facilitate language comprehension.

Of course, the situation can be different for less skilled readers, such as HSs who have difficulties with reading in a non-dominant language in general; as we found in Parshina et al. (2021a), they are slower, skip less and re-read more compared with their monolingual adult peers, much more resembling 9-year-old monolingual children or beginning L2 learners. The previous research with monolingual adults and children has repeatedly confirmed that literacy in general and reading skill in particular are important determinants of the predictive processing both in written and spoken language comprehension (Favier et al. 2021; Huettig and Pickering 2019, for review). Although HSs (and L2 learners) are highly literate in their dominant language, it is likely that lower levels of literacy experience with Russian diminish the ability to predict in the non-dominant language. 


\subsection{Present Study}

This study presents the first, novel systematic investigation of the online prediction abilities in heritage language reading. Specifically, we examine whether HSs of Russian can successfully generate full lexical and morphosyntactic predictions in the silent reading of simple isolated sentences in Russian. To that end, we conducted a secondary analysis of the previously collected eye-movement data from children and bilingual HSs and L2 learners of Russian (data from Parshina et al. 2021b). Our first two research questions follow from what we already know about reading in Russian as a heritage language (Parshina et al. 2021a, 2021b) and the recent utility model of prediction in L2 language comprehension (Kaan and Grüter 2021).

RQ1: Can English-dominant HSs of Russian anticipate lexical information (i.e., the upcoming words in the sentence)? Does the sensitivity of HSs to the lexical predictability differ from children or L2 learners?

RQ2: Can English-dominant HSs of Russian anticipate morphosyntactic information (i.e., the word class, noun gender, case and number and verb tense and number) and if yes, what types of morphosyntactic information (i.e., features) have the highest predictive value? Does the sensitivity of HSs to the morphosyntactic predictability differ from children or L2 learners?

We expect HSs to engage in both types of predictions similarly to monolingual children because HSs read on par with children (Parshina et al. 2021a) and in general show comparable language processing skills (Polinsky and Scontras 2020). It is likely, however, that the morphosyntactic features that have the highest predictive power are different in bilingual and monolingual reading. According to the utility account (Kaan and Grüter 2021), we expect that only noun number and verb tense will be used predictively and facilitate reading in non-dominant Russian for HSs (and L2 learners), as these features are (1) present and function as reliable predictive cues in the dominant English and thus can be transferred to the non-dominant language (Dussias et al. 2013; Hopp and Lemmerth 2018; van Bergen and Flecken 2017) and (2) transparent and salient features in Russian that are taught early in the L2 Russian classrooms and generally do not pose difficulties in heritage language acquisition (Benmamoun et al. 2013; Polinsky and Scontras 2020) or have already confirmed to be effective predictive cues in the spoken modality (e.g., Sekerina 2015).

Our last research question concerns the influence of the individual differences on the prediction ability:

RQ3: Do Russian literacy level or (oral) reading fluency in dominant English aid lexical or morphosyntactic prediction ability of bilingual readers?

Following the discussion in Huettig and Pickering (2019) for monolingual readers, we expect that HSs' ability to predict lexical information will be modulated by their levels of literacy experience with Russian. In particular, only HSs who have more experience with reading in Russian will be able to successfully anticipate the specific lexical item in its grammatical form. HSs, regardless of their Russian literacy levels and reading fluency, will be able to engage in morphosyntactic prediction as it is more common and more accessible (Luke and Christianson 2016) as well as more efficient in languages with rich morphology (Lopukhina et al. 2021).

To answer these research questions, we selected both early (first fixation duration [FFD], single fixation duration (SFD, i.e., duration of the first fixation in case the word receives one and only one fixation), gaze duration (GD, i.e., sum of all fixation durations in the first-pass reading), skipping probability) and late (total reading time (TT)) measures to see if they are affected by the lexical and morphosyntactic predictability of the words in the corpus. These measures are assumed to reflect the early (i.e., grapheme-to-phoneme decoding and early word recognition) and late stages (i.e., semantic and morphosyntactic integration to the context) of lexical processing, respectively (Rayner 2009). As we do not have the norming data for the words in the corpus from the HSs, we used the 
cloze probabilities obtained in a norming study with monolingual children (see Section 2 for details).

\section{Materials and Methods}

Previously, we collected the cloze probability data from monolingual adults. For this study, we also collected close probability norms from monolingual Russian-speaking children. The probabilities between adult and child groups across lexical items and all morphosyntactic features were strongly correlated (all $r s \geq 0.73, p s<0.001$ ), suggesting the cloze probabilities can be used interchangeably. As we mentioned above, the data from the previously published corpus study of eye movements of HSs in reading in Russian that we used here for our secondary analysis (Parshina et al. 2021b) do not contain cloze probability norms. Thus, in the absence of the cloze probability norms for Russian HSs, we opted to use the child-normed data to approximate word cloze probability in heritage language.

\subsection{Coze Probability Norming Study}

\subsubsection{Participants}

Forty-six Russian-speaking children (24 girls, $M_{\mathrm{age}}=11.3, S D=0.82$ and range 9-12) participated in the predictability norming study. All participants were recruited through social networks and the data collection was conducted online.

\subsubsection{Materials}

All materials were taken from the child version (Korneev et al. 2017) of the Russian Sentence Corpus (Laurinavichyute et al. 2019) and consisted of 30 sentences that were constructed to be read by primary-school typically developing Russian-speaking children (see Example (1) for illustration). Sentences represent diverse grammatical structures typical for Russian, namely, various word orders, active and passive voice constructions and various types of relative clauses. The mean length of the sentence was 8 words (range 6-9), the words were on average 5.6 letters long (range 1-13). All words were annotated for length and frequency (Lyashevskaya and Sharov 2009, the frequency was determined by means of the sub-corpus of texts for children of the years 1920-2015 of the National Corpus of the Russian Language at http:/ / ruscorpora.ru, accessed on 1 June 2017). The words were tagged for word class (nouns, verbs in finite forms, infinitives, adjectives, adverbs, numerals, personal pronouns, prepositions, conjunctions and particles), noun gender (masculine, feminine or neuter), noun number (singular or plural), noun case (nominative, genitive, accusative, dative, vocative and prepositional, including locative and ablative), verb tense (past, present or future) and verb number (singular or plural) using the PyMorphy2 analyzer (Korobov 2015).

$\begin{array}{llllll}\text { (1) В воскресенье } & \text { вся } & \text { дружная семья } & \text { поехала } & \text { на дачу. } \\ \text { On Sunday } & \text { all } & \text { friendly } & \text { family } & \text { went } & \text { to dacha } \\ \text { 'On Sunday, the whole friendly family went to the dacha.' } & & \end{array}$

\subsubsection{Procedure}

The task was to type a word that can be a natural continuation of the sentence, starting with a blank screen for the first word. After the response was provided, the actual target word was presented visually above the response box along with the prompt to type in the next word. Participants were not allowed to skip words or revisit previous responses, but they could exit the task at any point. Before data analysis, misprints or spelling mistakes were manually corrected, and the first words in all sentences were excluded from the analysis. In addition, we excluded data from participants who made guesses for fewer than 20 words.

Each response was binary coded (' 1 ' - the guess matched the target response, ' 0 ' the guess did not match). For lexical prediction, the response was coded as ' 1 ' only in the case when it fully matched the target word orthographically. For morphosyntactic prediction, each response was coded as ' 1 ' when it matched the target word in respective 
morphosyntactic features: word class, noun gender, case and number, as well as verb tense and number. For subsequent data analyses, cloze probabilities were log-transformed, zero cloze probabilities were replaced with $\frac{1}{2}{ }^{*}$ number of guesses for the word (Kliegl et al. 2004).

\subsection{Secondary Analysis of Eye Movements from the Previously Published Corpus}

Dataset

For all analyses, we used previously collected eye-movement data (Parshina et al. 2021b) freely available on the Open Science Framework project page (https:/ / osf.io/ek827, accessed on 25 February 2022). The participants read the same 30 sentences from the child version (Korneev et al. 2017) of the Russian Sentence Corpus (Laurinavichyute et al. 2019) that we used for the child norming study described in Section 2.1 above. The data came from 90 participants distributed in 3 groups, with 30 participants in each group: (1) Englishdominant HSs of Russian, (2) English-dominant L2 learners of Russian and (3) monolingual second graders (see Table 1 for participant characteristics).

Table 1. Participant characteristics and reading assessment scores.

\begin{tabular}{lccc}
\hline & HSs & L2 Learners & Children \\
\hline $\mathrm{N}$ & 30 & 30 & 30 \\
Women:Men & $14: 16$ & $21: 9$ & $11: 19$ \\
Age (range) & $17.5(13-24)$ & $21.2(16-43)$ & $8.5(8-9)$ \\
Age of Arrival to the USA & $3.9(4.8)$ & $0.1(0.7)$ \\
Oral Reading Fluency in English (ORF-Eng) & $26.6(6.3)$ & $27.6(5.5)$ \\
Literacy experience measures (Russian): & & & $18(5.6)$ \\
Age of reading start (years) & $8.4(5.1)$ & $8.3(2.7)$ \\
Oral Reading Fluency in Russian (ORF-Rus) & $12.3(6)$ & $2.6(0.9)$ \\
Self-estimated reading score (1-5) & $3(0.8)$ & $8.0(9.4)$ \\
Daily Russian exposure, including printed text (\%) & $25.6(18.6)$ & $0.894(0.91)$ \\
Russian literacy PC1 score & $-0.096(1.46)$ & \\
\hline
\end{tabular}

In addition to the eye-tracking reading task, the HS and L2 participants filled out the language background questionnaire which (among other questions) asked them to estimate their reading skills in Russian on a scale from 1 to 5 and the approximate age when they started to learn how to read in Russian. Furthermore, HS and L2 bilingual participants performed the Russian Oral Reading Fluency (ORF-Rus) and the English Oral Reading Fluency (ORF-Eng) tasks. The ORF-Rus test estimates the speed and the quality of reading out loud of the short (202 words) text in Russian, constructed for the neurolinguistic assessment of reading skills in 2nd-grade Russian-speaking children (Kornev 1997). The sentences in the text vary in complexity: they include a wide range of grammatical constructions typical for Russian as well as words of different frequencies.

The ORF-Eng test is the subtest of the standardized Woodcock Reading Mastery Tests (WRMT 3rd edition, Woodcock 2011). In the ORF-Eng test, participants read out loud the connected text (217 words) which contained English sentences of increasing difficulty. The average score (see Table 1) in both ORF-Rus and ORF-Eng tasks was calculated based on the formula that takes into account the time and number of errors readers made while reading the text (Woodcock Reading Mastery Tests formula, Woodcock 2011).

As the literacy measures for Russian were strongly intercorrelated, we performed a principal component analysis (using FactoMineR package, Lê et al. 2008) on the four measures (ORF-Rus, age of reading start, self-estimated reading competence and daily exposure to the Russian language). As a result, we derived the 'Russian literacy experience' variable (the first principal component, PC1) that explained 57\% of the variability in the data (Russian literacy PC1 score in Table 1, note that the low score indicates a higher Russian literacy level). All four measures contributed to the PC1, with the highest contribution by the ORF-Rus measure (28\%) and the lowest by the self-estimated reading competence measure $(23 \%)$. 


\section{Results}

\subsection{Cloze Probability Norming Study}

Table 2 presents mean cloze probabilities obtained in the cloze reading task by children. The results indicate that the morphosyntactic information (i.e., features of the words) was much more predictable than the lexical information (i.e., the specific lexical items and their grammatical forms). Specifically, the noun case and number were the most predictable morphosyntactic features, with the average accuracy of guessing at ceiling, followed by verb number and tense and noun gender. For example, children guessed the noun number (singular) and noun case (accusative) of the upcoming word dachu in Example (1) above with high accuracy (93\% and 98\%, respectively). The word class was the least predictable morphosyntactic feature. Finally, children were below chance in generating the full lexical prediction (see similar results in Lopukhina et al. 2021 for monolingual adults). For instance, even though being the final word in the sentence, the exact word dachu and its grammatical form (noun, feminine, singular and accusative) was fully predicted only with $6 \%$ accuracy in children.

Table 2. Mean cloze lexical and morphosyntactic probabilities (SDs).

\begin{tabular}{lc}
\hline & Cloze Probability \\
\hline Lexical Information & $0.163(0.063)$ \\
Morphosyntactic Information & \\
$\quad$ Word class & $0.546(0.110)$ \\
$\quad$ Nominal: & $0.913(0.090)$ \\
$\quad$ Case & $0.926(0.057)$ \\
Number & $0.708(0.090)$ \\
Gender & \\
Verbal: & $0.917(0.062)$ \\
$\quad$ Number & $0.853(0.113)$ \\
\hline Tense &
\end{tabular}

\subsection{Secondary Analysis of Eye Movements from the Previously Published Corpus}

We performed all data analyses in $\mathrm{R}$ (version 4.1.0; $\mathrm{R}$ Core Team 2021). Only the sentences with the correct answers to the comprehension questions were included in the analyses (HSs: $M_{\mathrm{acc}}=91 \%, S D=29 \%$; L2 learners: $M_{\mathrm{acc}}=85 \%, S D=36 \%$; Children: $M_{\text {acc }}=99 \%, S D=10 \%$ ). Fixations that were less than $100 \mathrm{~ms}$ were excluded as well as the first and last words in each sentence. Table 3 presents means and standard deviations for the dependent measures for all groups of readers.

Table 3. Means (SDs) for the FFD and skipping probability measures for three groups of readers.

\begin{tabular}{cccc}
\hline Measure & Children & L2 Learners & HSs \\
\hline FFD $(\mathrm{ms})$ & $316(114)$ & $358(99)$ & $361(146)$ \\
SFD $(\mathrm{ms})$ & $386(101)$ & $387(267)$ & $340(144)$ \\
GD $(\mathrm{ms})$ & $754(374)$ & $782(227)$ & $769(351)$ \\
TT $(\mathrm{ms})$ & $1066(588)$ & $1436(627)$ & $1210(577)$ \\
Skip $(\%)$ & $10(9)$ & $7(9)$ & $7(4)$ \\
\hline
\end{tabular}

We used (generalized) linear mixed models implemented through R (R Core Team 2021) package lme4 (1.1-13, Bates et al. 2015) and sjPlot package 2.8.3 (for data visualization and the computation of $p$-values; Lüdecke 2017). In all models, the outcome measures were:

1. First fixation duration (FFD);

2. Single fixation duration (SFD);

3. Gaze duration (GD);

4. Total reading time (TT);

5. The skipping probability of the target word. 
We ran a separate set of models for each outcome measure for investigating predictability of lexical and morphosyntactic information. All models included the following fixed predictors: the interaction between cloze probabilities (log-transformed) and group (treatment-coded, with HSs always being the reference level), centered and scaled word length, logarithm (base 10) of word frequency and relative position of the word in a sentence. The example of the $\mathrm{R}$ code for the model that estimates the impact of the lexical cloze probability on the FFD for HSs, L2 learners and children is in (2):

(2) $\log (\mathrm{FFD}) \sim$ frequency + length + relative position + group * lexical cloze probability +

(1 I participant $)+(1$ । sentence $)+(1$ । word $)$

For the bilingual groups (HSs and L2 learners), we also ran a set of models that estimated the series of the three-way interaction effects among the Russian literacy level (PC1 score), oral reading fluency in English (ORF-Eng, centered and scaled), group (deviation coded, ' 1 ' for HSs, ' -1 ' for L2 learners) and cloze probabilities. We also added an interaction term for the word frequency because it was reported to interact with predictability in L2 reading in the previous study (Mor and Prior 2021). The example of the $\mathrm{R}$ code for the model that estimated the impact of the word class cloze probability on the FFD for HSs and L2 learners is in (3):

(3) $\log ($ FFD $) ~$ length + relative position + word class cloze probability ${ }^{*}$ frequency ${ }^{*}$ group + word class cloze probability * $\mathrm{PC} 1^{*}$ group + word class cloze probability * ORF-Eng * group + (1 I participant $)+(1 \mid$ sentence $)+(1 \mid$ word $)$

The random predictors in all models included random intercepts for words, sentences, and participants (random intercept was removed in case the model failed to converge); all $p$-values were corrected using the Bonferroni-Holm correction. The full outputs for all (g)LMMs are located in the Supplementary Files with Tables S1-S6 at the OSF project page: https: / / osf.io/ek827, accessed on 25 February 2022. The full outputs with the results of the post-hoc simple slopes analyses for interaction effects are embedded as text in the R script that is freely available at the same page.

3.2.1. Can English-Dominant HSs of Russian Anticipate Lexical Information? Does the Sensitivity of HSs to the Lexical Predictability Differ from Children or L2 Learners?

For HSs, the effect of lexical predictability was significant in GD (Est. $=-0.103$, $S E=0.02, t=-4.33$ and $p<0.001)$ and TT eye-tracking measures $($ Est $=-0.162, S E=0.03$, $t=-6.37$ and $p<0.001$ ) (Table 4 ): the more predictable the lexical item was, the shorter the fixation durations. Following-up the group interactions in TT measure (L2: Est. $=0.08$, $S E=0.02, t=4.16$ and $p<0.001$; children: Est. $=0.10, S E=0.02, t=5.47$ and $p<0.001)$, the simple slopes analysis (using sim_slopes function in jtool R package function, Long 2019) showed that in TT measure, HSs differed from L2 and children in that the slope for the prediction effect was significantly steeper for the HSs, suggesting that in the late eyemovement measure, HSs were more sensitive to the lexical predictability than other groups (L2s: Est. $=-0.08, S E=0.03, t=-3.09$ and $p<0.001$; children: Est. $=-0.06, S E=0.02$, $t=-2.28$ and $p=0.020$ ) (Figure 1). In addition, the post-hoc simple slopes analysis for the interaction in the skipping probability $(O R=1.67, S E=0.27, z=3.13$ and $p=0.004)$ indicated that L2 learners, but not HSs, skipped more words with increasing lexical probability (Est. $=0.57, S E=0.16, z=3.52$ and $p<0.001$ ). Thus, we confirm that HSs do indeed engage in lexical prediction and are similar to children (and L2 learners) in sensitivity to the predictability effect in the early GD measure but show higher sensitivity in the later TT measure. 
Table 4. The (g)LMMs summary for the lexical probability (HSs is the reference group). Significant effects are highlighted in bold.

\begin{tabular}{|c|c|c|c|c|c|c|c|c|c|c|c|c|c|c|c|}
\hline \multirow[b]{2}{*}{ Predictors } & \multicolumn{3}{|c|}{ FFD } & \multicolumn{3}{|c|}{ SFD } & \multicolumn{3}{|c|}{ GD } & \multicolumn{3}{|c|}{ TT } & \multicolumn{3}{|c|}{ Skip } \\
\hline & Est. & $S E$ & $p$ & Est. & $S E$ & $p$ & Est. & $S E$ & $p$ & Est. & $S E$ & $p$ & Est. & $S E$ & $p$ \\
\hline (Intercept) & 5.498 & 0.065 & $<0.001$ & 5.564 & 0.061 & $<0.001$ & 6.048 & 0.083 & $<0.001$ & 6.585 & 0.101 & $<0.001$ & 0.021 & 0.007 & $<0.001$ \\
\hline Frequency & -0.042 & 0.009 & $<0.001$ & -0.055 & 0.011 & $<0.001$ & -0.114 & 0.012 & $<0.001$ & -0.148 & 0.013 & $<0.001$ & 1.373 & 0.097 & $<0.001$ \\
\hline Length & 0.041 & 0.012 & 0.002 & -0.032 & 0.016 & 0.112 & 0.258 & 0.016 & $<0.001$ & 0.283 & 0.019 & $<0.001$ & 0.523 & 0.054 & $<0.001$ \\
\hline Group [L2] & 0.058 & 0.077 & 0.453 & 0.106 & 0.063 & 0.164 & 0.134 & 0.097 & 0.216 & 0.334 & 0.121 & 0.006 & 1.164 & 0.366 & 0.642 \\
\hline Group [CH] & -0.058 & 0.076 & 0.453 & -0.043 & 0.063 & 0.556 & 0.051 & 0.097 & 0.595 & 0.034 & 0.120 & 0.781 & 1.187 & 0.364 & 0.642 \\
\hline Lexical probability & -0.042 & 0.023 & 0.128 & -0.018 & 0.023 & 0.533 & -0.103 & 0.024 & $<0.001$ & -0.161 & 0.025 & $<0.001$ & 1.068 & 0.151 & 0.642 \\
\hline L2* Lexical probability & 0.026 & 0.027 & 0.453 & -0.010 & 0.024 & 0.685 & 0.034 & 0.022 & 0.211 & 0.081 & 0.020 & $<0.001$ & 1.664 & 0.271 & 0.004 \\
\hline $\mathrm{CH}^{*}$ Lexical probability & 0.022 & 0.026 & 0.453 & -0.021 & 0.023 & 0.533 & 0.031 & 0.021 & 0.211 & 0.104 & 0.019 & $<0.001$ & 0.831 & 0.109 & 0.239 \\
\hline \multicolumn{16}{|l|}{ Random effects } \\
\hline$\sigma^{2}$ & & 0.24 & & \multicolumn{3}{|c|}{0.22} & \multicolumn{3}{|c|}{0.38} & \multicolumn{3}{|c|}{0.30} & \multicolumn{3}{|c|}{3.29} \\
\hline \multirow{3}{*}{$\tau_{00}$} & \multicolumn{3}{|c|}{$0.01_{\text {word }}$} & \multicolumn{3}{|c|}{$0.01_{\text {word }}$} & \multicolumn{3}{|c|}{$0.01_{\text {word }}$} & \multicolumn{3}{|c|}{$0.02_{\text {word }}$} & \multicolumn{3}{|c|}{$0.28_{\text {word }}$} \\
\hline & \multirow{2}{*}{\multicolumn{3}{|c|}{$\begin{array}{c}0.06_{\text {subject }} \\
0.00_{\text {item }}\end{array}$}} & \multirow{2}{*}{\multicolumn{3}{|c|}{$\begin{array}{c}0.05_{\text {subject }} \\
0.00_{\text {item }}\end{array}$}} & \multirow{2}{*}{\multicolumn{3}{|c|}{$\begin{array}{c}0.13_{\text {subject }} \\
0.00_{\text {item }}\end{array}$}} & \multirow{2}{*}{\multicolumn{3}{|c|}{$\begin{array}{c}0.21_{\text {subject }} \\
0.01_{\text {item }}\end{array}$}} & \multirow{2}{*}{\multicolumn{3}{|c|}{$\begin{array}{c}1.05_{\text {subject }} \\
0.08_{\text {item }}\end{array}$}} \\
\hline & & & & & & & & & & & & & & & \\
\hline Observations & \multicolumn{3}{|c|}{7008} & \multicolumn{3}{|c|}{5613} & \multicolumn{3}{|c|}{12,709} & \multicolumn{3}{|c|}{12,709} & \multicolumn{3}{|c|}{13,780} \\
\hline $\mathrm{R} 2 / \Omega 02$ & \multicolumn{3}{|c|}{$0.029 / 0.243$} & \multicolumn{3}{|c|}{$0.031 / 0.224$} & \multicolumn{3}{|c|}{$0.216 / 0.429$} & \multicolumn{3}{|c|}{$0.287 / 0.600$} & \multicolumn{3}{|c|}{$0.211 / 0.446$} \\
\hline
\end{tabular}




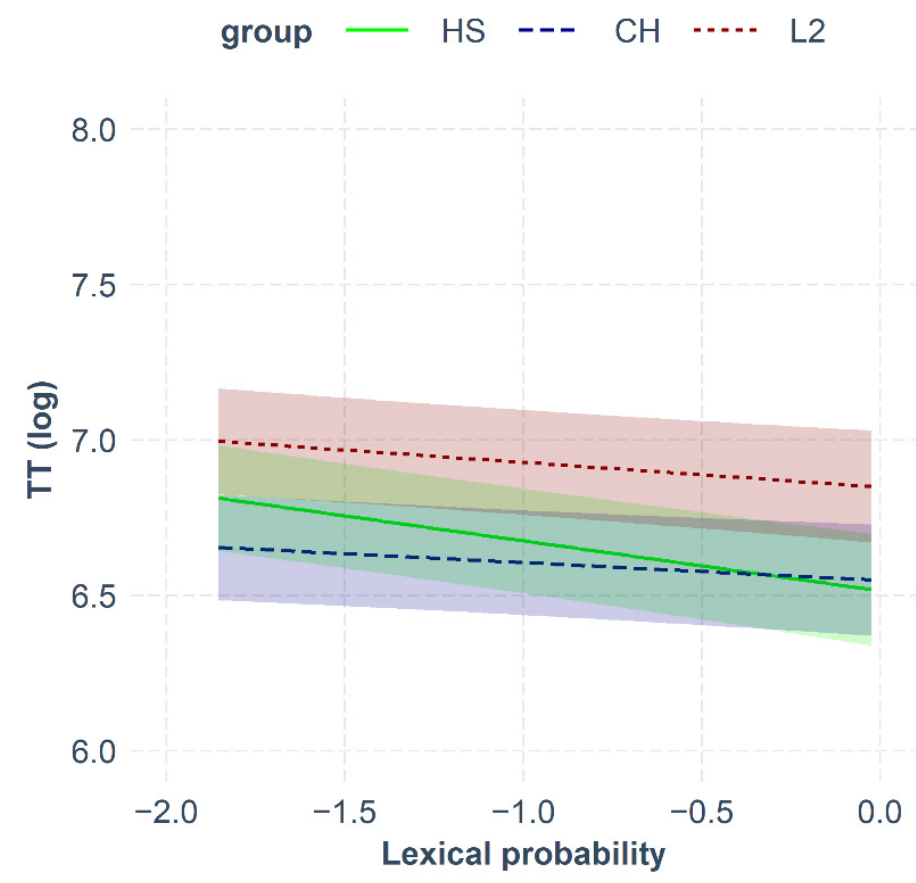

Figure 1. Interaction plot for the TT duration measure as a function of the lexical probability.

3.2.2. Can English-Dominant HSs of Russian Anticipate Morphosyntactic Information and If Yes, What Morphosyntactic Features Have the Highest Predictive Value? Does the Sensitivity of HSs to Morphosyntactic Predictability Differ from Children or L2 Learners?

Again, as with the effect of predictability on lexical information (Section 3.2.1), the same two eye-tracking measures, i.e., GD and TT, revealed that HSs were sensitive to the word class predictability (Est. $=-0.090, S E=0.02, t=-3.82$ and $p=<0.001 ;$ Est. $=-0.095$, $S E=0.04, t=-2.66$ and $p=0.014$, respectively) (Table S1 in Supplementary Files), with shorter fixation durations on the word that had higher word class predictability. Following up the group interactions in TT measure (Est. $=0.07, S E=0.03, t=2.53$ and $p=0.013$ ), the simple slopes analysis indicated that there was no effect of the word class probability for L2 learners (Est. $=-0.02, S E=0.04, t=-0.61$ and $p=0.54$ ) (Figure 2).

Among nominal morphosyntactic features (Table S2: noun gender, case and number), the only effect that was present was the effect of the noun number predictability in TT measure (increasing noun number cloze probability resulted in decreasing fixation duration, Est. $=-0.41, S E=0.17, t=-2.41$ and $p=0.035$ ). Following up on the significant interactions in TT measure between noun number predictability and group (children: Est. $=0.42$, $S E=0.12, t=3.45$ and $p=0.002$; L2: Est. $=0.32, S E=0.14, t=2.32$ and $p=0.038$ ), the posthoc simple slope analysis indicated that there was no effect of noun number for children (Est. $=0.01, S E=0.17, t=0.04$ and $p=0.097$ ) or L2 learners (Est. $=-0.10, S E=0.18, t=-0.54$ and $p=0.059)$. Children were sensitive to the noun gender predictability in TT measure (Est. $=-0.29, S E=0.10, t=2.76$ and $p=0.014$ ): the post-hoc analysis revealed that for them, the total reading times for words decreased with increasing noun gender probability (Est. $=-0.30, S E=0.14, t=-2.20$ and $p=0.030$ ).

Finally, with increasing verb number predictability (Table S3), HSs had shorter fixation durations in GD and TT measures (GD: Est. $=-0.359, S E=0.13, t=-2.77$ and $p=0.017$; TT: Est. $=-0.352 S E=0.14, t=-2.60$ and $p=0.022$ ). There were no significant interaction effects with the other groups. In sum, we confirmed that HSs anticipated the morphosyntactic features of the words in early GD and late TT measures and were similar to children in sensitivity to the word class and verb number predictability. HSs differed from children (and L2 learners) in prediction of the nominal features. 


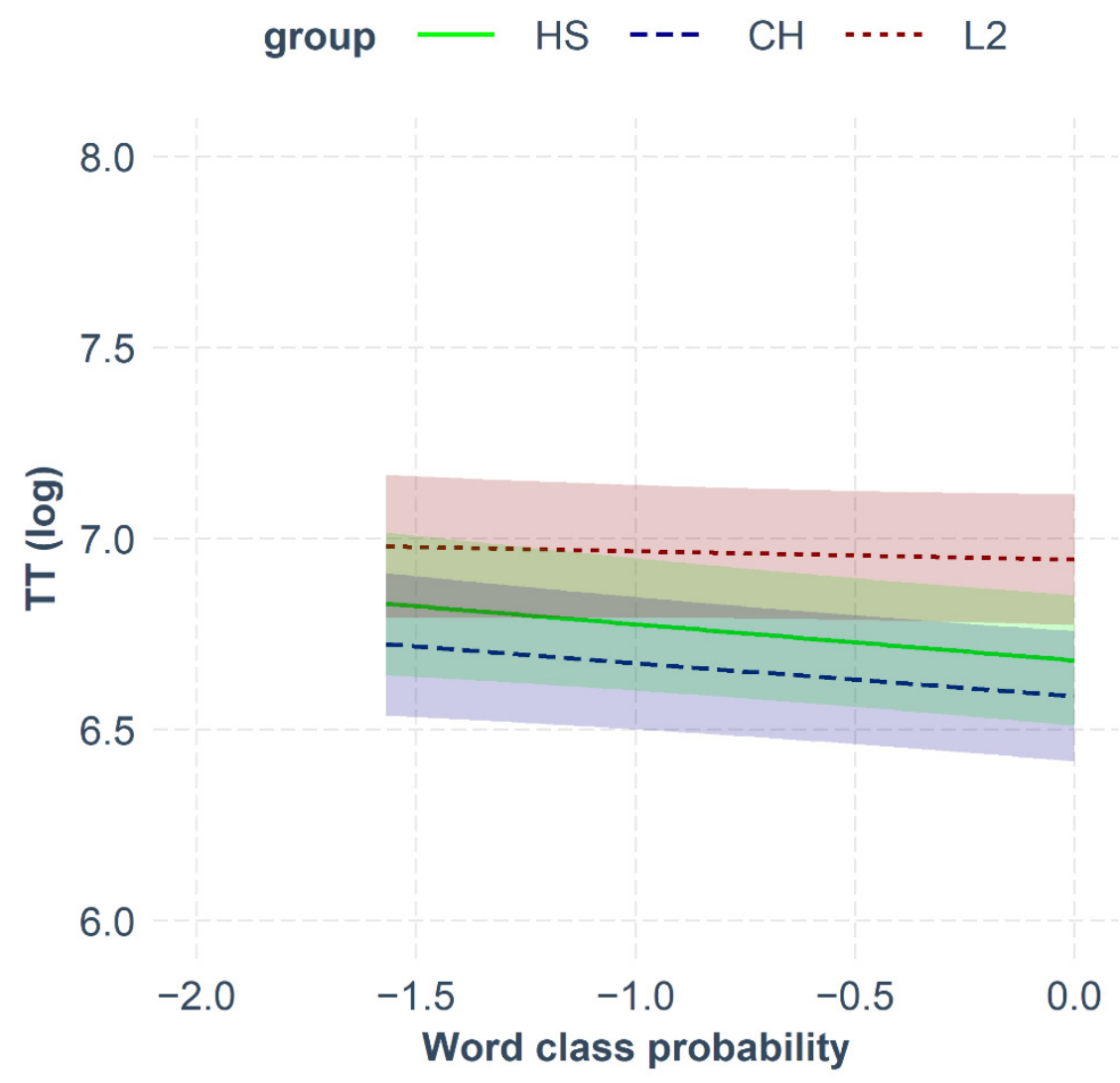

Figure 2. Interaction plot for the TT duration measure as a function of the word class probability.

3.2.3. Do Russian Literacy Level or (Oral) Reading Fluency in Dominant English Aid Lexical or Morphosyntactic Prediction Ability of Bilingual Readers?

Individual Differences and Lexical Predictability

The first thing to mention is we found strong effects of the levels of literacy experience with Russian (PC1) for both groups in all fixation duration measures (FFD, SFD, GD and TT) and skipping probability (Table S4). The lower level of literacy was associated with the longer fixation durations and lower rates of skipping the words (all $p s<0.001$ ). There were, however, significant interaction effects between PC1 and the lexical predictability in SFD (Est. $=0.03, S E=0.12, t=2.48$ and $p=0.034), \mathrm{TT}(E s t .=-0.04, S E=0.01, t=4.39$ and $p<0.001)$ and skipping probability $(O R=0.78, S E=0.07, z=-2.76$ and $p=0.021)$ measures. The post-hoc simple slopes analysis with set values at 1 SD below and above the PC1 mean suggested that with increasing lexical predictability, participants with a higher literacy level (at -1 SD) fixated words for shorter times in SFD measure $(E s t .=-0.08, S E=0.03$, $t=-2.64$ and $p=0.010)$. When the literacy level was lower ( $1 \mathrm{SD}$ above the mean), the slope for the prediction effect was non-significant (Est. $=0.02, S E=0.03, t=0.66$ and $p=0.510$ ). In TT measure, however, only the participants with lower Russian literacy scores (at +1 SD) were sensitive to the lexical predictability of the words, with shorter fixations on the words that had higher lexical predictability (Est. $=-0.16, S E=0.03, t=-4.96$ and $p<0.001)$. Finally, in skipping probability, we found that the participants with lower Russian literacy level skipped fewer words as the lexical predictability became higher (Est. $=-0.48, S E=0.23, z=-2.15$ and $p=0.030$ ).

The oral reading fluency in English alone did not affect any of the dependent measures, but there was an interaction with lexical predictability in TT measure $(E s t .=-0.03$, $S E=0.01, t=2.64$ and $p=0.015$ ): Those who performed better in the ORF-Eng task (at $1 \mathrm{SD}$ above the mean) fixated on highly predictable words for shorter times than those who received lower scores (at $-1 \mathrm{SD})($ sst. $=-0.12, S E=0.03, t=-3.89$ and $p<0.001$; Est. $=-0.07, S E=0.03, t=-2.16$ and $p<0.003$, respectively). 
Individual Differences and Morphosyntactic Predictability

We found the interaction effect (Table S5) between the word class predictability and the Russian literacy in the GD measure (Est. $=-0.04, S E=0.02, t=-2.28$ and $p=0.033$ ). The follow-up post-hoc simple slopes analysis on this interaction indicated that bilinguals with lower Russian literacy level (at $+1 \mathrm{SD}$ ) fixated words with higher word class predictability for shorter times (Est. $=-0.12, S E=0.03, t=-4.03$ and $p<0.001$ ), whereas participants with better Russian literacy skills $(-1 \mathrm{SD})$ were not sensitive to the word class predictability (Est. $=-0.00, S E=0.04, t=-0.06$ and $p=0.095$ ). In addition, the interaction effect in the FFD measure between group, Russian literacy and word class probability predictors (Est. $=-0.04, S E=0.02, t=-2.38$ and $p=0.045$ ) suggested that L2 learners with better Russian literacy skills (but not HSs) produced shorter first fixation durations on words with higher word class predictability (Est. $=-0.16, S E=0.07, t=-2.23$ and $p=0.03$ ).

We also tested the interaction effects of the individual difference factors with nominal and verbal morphosyntactic predictability for the eye-movement measures that were influenced in the group comparison models, but there were none (Table S6). In sum, we confirmed the impact of the Russian literacy experience and (oral) reading fluency in dominant English on the lexical and morphosyntactic word class predictability, but the expected effect was evident only in some of the eye-tracking measures.

\section{Discussion}

In this study, we investigated the ability of bilingual HSs to predict lexical information (i.e., anticipate the upcoming word forms) and/or the morphosyntactic features of the words in reading isolated sentences in non-dominant Russian. In general, we established that HSs (and L2 learners) indeed pre-activate lexical and morphosyntactic features of the words as evident in early and late eye-movement measures. Our general hypothesis was that HSs would resemble children in their prediction ability and indeed, in the early GD measure, all the effects (or their absence) were similar between the groups. In the late TT measure, HSs were more sensitive to the lexical predictability of the words than children and HSs were the only group who showed prediction of the noun number feature. Notably, we also observed similar predictive preferences and trends between HSs and L2 learners, although for the latter group the effects were prevalent in the early eye-tracking measures (FFD, GD and skipping probability).

Finally, we partially confirmed our hypotheses concerning the influence of the levels of literacy experience with Russian on the ability to predict-higher levels of Russian literacy experience aided prediction as evident in the earliest eye-tracking measures. In a later measure (TT), however, higher lexical and morphosyntactic predictability was only beneficial for HSs (and L2 learners) with less Russian literacy experience. Below, we discuss the findings in more detail with respect to our research questions articulated in Section 1.4.

4.1. RQ1: Can English-Dominant HSs of Russian Anticipate Lexical Information (i.e., the Upcoming Words in the Sentence)? Does the Sensitivity of HSs to the Lexical Predictability Differ from Children or L2 Learners?

To begin with, we hypothesized that HSs would be able to engage in lexical prediction (modulated by the Russian literacy levels) and that they would resemble children in this ability. The expectations were based on previously reported similarities in eye-movement characteristics in reading among HSs and children (Parshina et al. 2021a, 2021b) and theoretical accounts that suggest similar language development trajectories of these groups (see the discussion of the divergent attainment trajectory, Scontras et al. (2015)). For children, our analyses show that the increase in lexical cloze probabilities decreases the gaze duration and total reading times of the words even though the accuracy of the lexical prediction in the cloze probability norming study by children reached only $16 \%$ (and 18\% in monolingual adults in Lopukhina et al. 2021). In line with findings in Lopukhina et al. (2021) and Luke and Christianson (2016), these results indicate that although young and adult 
monolingual speakers are able to anticipate the specific lexical item in reading to a certain extent, this type of prediction does not occur frequently and does not reach high accuracy.

We only partially confirmed the general hypothesis of similarities between HSs and children. In early GD measure, the sensitivity to the lexical cloze probabilities of the words did not differ, not only between HSs and children, but also between HSs and L2 learners. However, in late TT measure, HSs were considerably more sensitive to the change in the lexical predictability of the words, as the words with higher lexical predictability received overall shorter reading times. This difference suggests that HSs may be more effective in lexical prediction than their 9-year-old peers, at least at the 'later' stages of the semantic and morphosyntactic information integration. Future studies, however, should investigate the question further, starting with the direct comparison of cloze probabilities obtained from the HSs and children.

4.2. RQ2: Can English-Dominant HSs of Russian Anticipate Morphosyntactic Information and If Yes, What Morphosyntactic Features Have the Highest Predictive Value? Does the Sensitivity of HSs to the Morphosyntactic Predictability Differ from Children or L2 Learners?

Among the morphosyntactic features we investigated (word class, noun gender, case and number, as well as verb number and tense), only a few had the predictive value in reading in heritage language but those few showed a strong effect. In early GD and late TT measures, similar to children and L2 learners (in GD only), HSs successfully predicted the upcoming word class. HSs were the only ones to rely on the noun number predictability (in TT measure), but the three groups (HSs, L2 learners and children) were equally sensitive to the verb number predictability (GD and TT). Finally, the children group was the only group who produced shorter fixation durations (in TT) with increasing noun gender predictability, on par with the monolingual adults in the previous study by Lopukhina et al. (2021).

In general, our results for monolingual children support previous findings in Lopukhina et al. (2021) for monolingual adults that such features as noun case and number, despite being the most predictable features as assessed through the cloze task, do not affect the reading speed or skipping probability for children. Lopukhina and colleagues suggest two possible explanations. First, it is likely that the morphosyntactic predictability of some features does not account for enough variance in the eye-movement behavior in reading in comparison with other well-known factors such as word length, word frequency or relative position of the word in a sentence (see Staub and Rayner 2007, for review). Another possibility is that morphosyntactic cues weigh differently in comprehension and production. For example, in the cloze task in our study, the noun case and number were highly predictable for children, but they did not affect eye-movement measures in reading. Word class and noun gender, on the other hand, were the least predictable features in the cloze task, but they reliably facilitated reading.

So where do HSs stand on the morphosyntactic prediction in reading in Russian? Recall that our expectations for their ability to anticipate the morphosyntactic information were based on the knowledge that morphosyntactic prediction is a more common and more efficient type of prediction (Luke and Christianson 2016), including prediction in reading in Russian (Lopukhina et al. 2021), and thus should be accessible to HSs (and L2 learners) even before the full lexical prediction. However, following the utility account, we hypothesized that not all, but only those morphosyntactic features that serve as reliable predictive cues in the "cost-benefit" analysis (Kaan and Grüter 2021), will affect reading speed and skipping rate in bilingual reading. The reliable predictive features are the ones that can be transferred from dominant English (Foucart 2021), namely noun number (singular/plural) and verb tense (past/present/future). These features are reported to not pose many difficulties in heritage language development (Polinsky and Scontras 2020 for discussion of the verb tense). In addition, there is already some evidence of the number feature functioning as a predictive cue in heritage language processing (Sekerina 2015).

First, we indeed found that both HSs and L2 learners were sensitive to the predictability of some morphosyntactic features, supporting the views that such partial prediction is accessible for bilinguals, and it facilitates language comprehension (e.g., Dussias et al. 2013; 
Hopp 2021; Morales et al. 2016; Schlenter and Felser 2021). However, except for HSs who were sensitive to the noun number predictability in the late processing stages (TT), we did not observe any verb tense predictability effects. Instead, similar to children, HSs and L2 learners successfully pre-activated the verb number information, which is largely absent in dominant English. We can speculate, therefore, that for these groups, agreement between nouns and verbs in Russian is reliable and perceptually salient enough to have a facilitative effect on the eye movements in reading, even though the feature is not present in L1. It may be because the agreement morphology in Russian, especially in number, is the most accessible type for acquisition (and quantification in general; Gor 2019; Sekerina 2015). In addition, the verbal agreement in heritage languages is believed to be more resilient to attrition in comparison with noun agreement (Polinsky 2018, for review; Polinsky 2006).

We also found that similar to children (in GD and TT) and L2 learners (in GD), the fixation durations for HSs were shorter for the words with high word class cloze probability. In L2 learners, the same effect was present for the first fixation duration but only for those participants who had a high Russian literacy level. We suggest that the results indicate that the word class predictive cues might also be transferrable across languages but as a generic representation or general cognitive skill. Bilinguals are 'trained' to predict the upcoming word class in dominant English based on the word order and the context of the sentence (Luke and Christianson 2016). It is likely that the skill is also implemented in reading in the non-dominant Russian, taken that the reading skill is developed in general and in Russian in particular.

4.3. RQ3: Do Russian Literacy Level or (Oral) Reading Fluency in Dominant English Aid Lexical or Morphosyntactic Prediction Ability of Bilingual Readers?

In some of the early eye-tracking measures (SFD and FFD), our results for lexical and morphosyntactic (word class) predictability were contingent on the degree of the Russian literacy experience; participants with more Russian literacy experience fixated the words with higher cloze probability for shorter times. The results are consistent with the discussion of the role that literacy plays in the prediction in Huettig and Pickering (2019). More skilled readers experience benefits in prediction from the primary reading influences (the 'trained' eye-movement behavior in reading, e.g., landing the saccade in the optimal viewing position (O'Regan and Jacobs 1992)) and secondary influences (e.g., greater vocabulary knowledge and working memory). In addition, we also found that sensitivity to lexical predictability for HSs and L2 learners depends on the oral reading fluency in dominant English (in TT measure), which can indicate that the primary and some of the secondary literacy influences on prediction are transferrable across languages, even when languages do not share the script (see Kuperman et al. (2020) for the discussion of the similarities in L1 and L2 eye-movement reading behavior, but cf. Mor and Prior 2021). Except for the word class, we did not observe the impact of the Russian literacy experience on the predictability effects in any other morphosyntactic features.

We did, however, find somewhat unexpected trends in lexical predictability and word class predictability in other eye-tracking measures (TT and GD measures, respectively): the Russian literacy skills aided the prediction but only for participants with less Russian literacy experience. We suggest that for our bilingual participants, greater literacy in the non-dominant language is especially beneficial for prediction at the earliest stages of word recognition when the word is fixated for the first time (FFD and SFD), as these readers are likely to experience fewer difficulties with the initial lexical access. At the same time, the prediction ability is advantageous at later stages of lexical access (GD) and post-lexical information integration (TT), but only for bilinguals with less exposure to written materials in Russian, after the grapheme-phoneme conversion process is completed and all initial difficulties are resolved. We also saw that these bilinguals skipped fewer words as lexical predictability increased. We can speculate that for readers who have less experience in reading in the second language, more predictable words are also more recognizable, and thus the readers 'choose' to fixate them for further lexical processing. 
In contrast, words with lower predictability are skipped as a possible strategy to avoid lexical processing and as an attempt to derive the meaning of the word from contextual information. This hypothesis is supported by the interaction between word frequency and lexical predictability that we found in our study in the early SFD measure and skipping probability (the latter was marginally significant at $p=0.056$, see Table S4). Specifically, with increasing lexical predictability, bilinguals fixated only more frequent words for a shorter time and skipped less the low-frequency words (cf. Mor and Prior who found L2 readers skipped less low-frequency words that were also less predictable).

\subsection{Conclusions}

Ultimately, our findings support the predictions of the utility account (Kaan and Grüter 2021), according to which the bilinguals who have more non-dominant language experience and for whom the reading task is not too resource-demanding, should 'opt' to engage in lexical prediction while reading, starting from the early stages of the word recognition (see Whitford and Titone 2017 for similar results). In morphosyntactic prediction, the results also speak in favor of the utility account but perhaps with a more complex system of weighing the reliability of the predictive cues in heritage language than we initially anticipated. For HSs, the cost-benefit analysis likely includes not only the factors of the cue transferability, reliability, the task demands and the proficiency level, but also the reliability of the cues within the heritage language grammar per se, and those can differ from one heritage speaker to another (Polinsky 2018; Scontras et al. 2015) and heavily depend on the frequency and regularity of the cues occurring in the heritage input. Future studies, therefore, should focus on the investigation of the predictive values of various cues (lexical or morphosyntactic) solely based on the input provided by HSs.

In our study, we also saw a lot of similarities in the predictive preferences between HSs and children in the early eye-tracking measures and some differences in the late total reading time measure (i.e., greater sensitivity to lexical predictability and noun gender vs. noun number predictability effects). These results, while providing further evidence for similar reading behavior of these groups at the early stages of lexical access (as predicted by the divergent attainment trajectory, Polinsky and Scontras (2020)), also indicate that prediction facilitates the stages of semantic and syntactic integration more for HSs than for children and that the facilitative use of some predictive morphosyntactic cues is different for these groups. The explanation may be intuitive: HSs have more experience with reading materials (in English and Russian combined) and they can experience the cross-language transfer effects.

In comparison with L2 learners, HSs in general also showed more sensitivity to lexical and morphosyntactic predictability in late TT measure, whereas L2 learners (with higher Russian literacy level) had more effects in early eye-tracking measures. Future studies should further examine whether it is the manner of language acquisition that led to these differences: could early exposure to the spoken language that HSs receive in early childhood lead to 'better' prediction abilities that aid semantic and syntactic information integration in sentence reading? Or could L2 learners' formal instruction in the nondominant language (including reading and writing) help develop prediction abilities that aid the earliest stages of word recognition in reading? We believe that exploring these questions will provide researchers with more insights about bilingual prediction, including the four central questions of language prediction (Huettig 2015): 'why', 'what', 'how' and 'when' do readers predict the upcoming information.

Supplementary Materials: The following supporting information can be downloaded at: https: //www.mdpi.com/article/10.3390/languages7010060/s1.

Author Contributions: Conceptualization, O.P., A.L. and I.A.S.; methodology, O.P. and A.L.; software, O.P. and A.L.; validation, O.P., A.L. and I.A.S.; formal analysis, O.P.; investigation, O.P., A.L. and I.A.S.; resources, O.P. and A.L.; data curation, O.P. and A.L.; writing-original draft preparation, O.P.; writing-review and editing, O.P., A.L. and I.A.S.; visualization, O.P.; supervision, I.A.S.; project 
administration, O.P.; funding acquisition, O.P., A.L. and I.A.S. All authors have read and agreed to the published version of the manuscript.

Funding: This study was performed with financial support from the RF Government Grant, ag. No. 14.641.31.0004.

Institutional Review Board Statement: The study was conducted according to the guidelines of the Declaration of Helsinki, and approved by the Ethics Committee of the HSE University $(11 / 10 / 2020)$.

Informed Consent Statement: Informed consent was obtained from all subjects involved in the study.

Data Availability Statement: Data and R script can be found at the OSF project page: https:/ / osf.io/ ek827.

Conflicts of Interest: The authors declare no conflict of interests.

\section{References}

Balota, David A., Alexander Pollatsek, and Keith Rayner. 1985. The interaction of contextual constraints and parafoveal visual information in reading. Cognitive Psychology 17: 364-90. [CrossRef]

Bates, Douglas, Martin Mächler, Ben Bolker, and Steve Walker. 2015. Fitting Linear Mixed-Effects Models Using lme4. Journal of Statistical Software 67: 1-148. [CrossRef]

Benmamoun, Elabbas, Silvina Montrul, and Maria Polinsky. 2013. Heritage languages and their speakers: Opportunities and challenges for linguistics. Theoretical Linguistics 39: 129-81. [CrossRef]

Boston, Marisa Ferrara, John Hale, Reinhold Kliegl, Umesh Patil, and Shravan Vasishth. 2008. Parsing costs as predictors of reading difficulty: An evaluation using the Potsdam Sentence Corpus. Journal of Eye Movement Research 2: 1-12. [CrossRef]

Demberg, Vera, and Frank Keller. 2008. Data from eye-tracking corpora as evidence for theories of syntactic processing complexity. Cognition 109: 193-210. [CrossRef]

Dussias, Paola E., Jorge R. Valdés Kroff, Rosa E. Guzzardo Tamargo, and Chip Gerfen. 2013. When gender and looking go hand in hand: Grammatical Gender Processing In L2 Spanish. Studies in Second Language Acquisition 35: 353-87. [CrossRef]

Favier, Saoradh, Antje S. Meyer, and Falk Huettig. 2021. Literacy can enhance syntactic prediction in spoken language processing Journal of Experimental Psychology: General 150: 2167-74. [CrossRef]

Foucart, Alice. 2021. Language prediction in second language: Does language similarity matter? In Prediction in Second Language Processing and Learning. Edited by E. Kaan and T. Grüter. Amsterdam: John Benjamins Publishing Company, vol. 12, pp. 92-114. [CrossRef]

Foucart, Alice, Clara D. Martin, Eva M. Moreno, and Albert Costa. 2014. Can bilinguals see it coming? Word anticipation in L2 sentence reading. Journal of Experimental Psychology: Learning, Memory, and Cognition 40: 1461-69. [CrossRef]

Foucart, Alice, Elisa Ruiz-Tada, and Albert Costa. 2016. Anticipation processes in L2 speech comprehension: Evidence from ERPs and lexical recognition task. Bilingualism: Language and Cognition 19: 213-19. [CrossRef]

Frenck-Mestre, Cheryl, Seung Kyung Kim, Hyeree Choo, Alain Ghio, Julia Herschensohn, and Sungryong Koh. 2019. Look and listen! The online processing of Korean case by native and non-native speakers. Language, Cognition and Neuroscience 34: 385-404. [CrossRef]

Fuchs, Zuzanna. 2021. Facilitative use of grammatical gender in Heritage Spanish. Linguistic Approaches to Bilingualism. [CrossRef]

Gollan, Tamar H., Timothy J. Slattery, Diane Goldenberg, Eva Van Assche, Wouter Duyck, and Keith Rayner. 2011. Frequency drives lexical access in reading but not in speaking: The frequency-lag hypothesis. Journal of Experimental Psychology: General 140: 186-209. [CrossRef] [PubMed]

Gor, Kira. 2019. Morphosyntactic Knowledge in Late Second Language Learners and Heritage Speakers of Russian. Heritage Language Journal 16: 124-50. [CrossRef]

Grüter, Theres, Casey Lew-Williams, and Anne Fernald. 2012. Grammatical gender in L2: A production or a real-time processing problem? Second Language Research 28: 191-215. [CrossRef] [PubMed]

Henry, Nick, Carrie N. Jackson, and Holger Hopp. 2020. Cue coalitions and additivity in predictive processing: The interaction between case and prosody in L2 German. Second Language Research, 026765832096315. [CrossRef]

Hopp, Holger. 2013. Grammatical gender in adult L2 acquisition: Relations between lexical and syntactic variability. Second Language Research 29: 33-56. [CrossRef]

Hopp, Holger. 2015. Semantics and morphosyntax in predictive L2 sentence processing. International Review of Applied Linguistics in Language Teaching 53: 277-306. [CrossRef]

Hopp, Holger. 2021. Prediction and grammatical learning in second language sentence processing. In Prediction in Second Language Processing and Learning. Edited by E. Kaan and T. Grüter. Amsterdam: John Benjamins Publishing Company, pp. 168-85.

Hopp, Holger, and Natalia Lemmerth. 2018. Lexical and syntactic congruency in predictive gender processing. Studies in Second Language Acquisition 40: 171-99. [CrossRef]

Huettig, Falk. 2015. Four central questions about prediction in language processing. Brain Research 1626: 118-35. [CrossRef] 
Huettig, Falk, and Martin J. Pickering. 2019. Literacy advantages beyond reading: Prediction of spoken language. Trends in Cognitive Sciences 23: 464-75. [CrossRef]

Ito, Aine, Andrea E. Martin, and Mante S. Nieuwland. 2017. On predicting form and meaning in a second language. Journal of Experimental Psychology: Learning, Memory, and Cognition 43: 635-52. [CrossRef]

Ito, Aine, and Martin J. Pickering. 2021. Automaticity and prediction in non-native language comprehension. In Prediction in SecondLanguage Processing and Learning. Edited by E. Kaan and T. Grüter. Amsterdam: John Benjamins Publishing Company, pp. 26-46. [CrossRef]

Kaan, Edith. 2014. Predictive sentence processing in L2 and L1: What is different? Linguistic Approaches to Bilingualism 4: 257-82. [CrossRef]

Kaan, Edith, and Theres Grüter. 2021. Prediction in second language processing and learning. Advances and Directions. In Prediction in Second Language Processing and Learning. Edited by E. Kaan and T. Grüter. Amsterdam: John Benjamins Publishing Company, pp. 2-24. [CrossRef]

Kliegl, Reinhold, Ellen Grabner, Martin Rolfs, and Ralf Engbert. 2004. Length, frequency, and predictability effects of words on eye movements in reading. European Journal of Cognitive Psychology 16: 262-84. [CrossRef]

Korneev, Aleksei, Ekaterina Matveeva, and Tat'yana Akhutina. 2017. Silent reading in Russian primary schoolchildren: An eye tracking study. Psychology. Journal of the Higher School of Economics 14: 219-35. [CrossRef]

Kornev, A. N. 1997. Нарушение чтения и письма у детей: Учебно-методическое пособие [Reading and Writing Disorders in Children: Study Guide]. Saint Petersburg: M and M.

Korobov, Mikhail. 2015. Morphological Analyzer and Generator for Russian and Ukrainian Languages. In Analysis of Images, Social Networks and Texts. Edited by M. Yu Khachay, N. Konstantinova, A. Panchenko, D. Ignatov and V. G. Labunets. Cham: Springer International Publishing, pp. 320-32.

Kuperberg, Gina R., and T. Florian Jaeger. 2016. What do we mean by prediction in language comprehension? Language, Cognition and Neuroscience 31: 32-59. [CrossRef]

Kuperman, Victor, Noam Siegelman, Sascha Schroeder, Cengiz Acartürk, Svetlana Alexeeva, Simona Amenta, Raymond Bertram, Rolando Bonandrini, Marc Brysbaert, Daria Chernova, and et al. 2020. Text reading in English as a second language: Evidence from the multilingual eye-movements corpus (MECO). Studies in Second Language Acquisition, Manuscript submitted for publication.

Laurinavichyute, Anna K., Irina A. Sekerina, Svetlana Alexeeva, Kristine Bagdasaryan, and Reinhold Kliegl. 2019. Russian Sentence Corpus: Benchmark measures of eye movements in reading in Russian. Behavior Research Methods 51: 1161-78. [CrossRef]

Lê, Sébastien, Julie Josse, and François Husson. 2008. FactoMineR: An R Package for Multivariate Analysis. Journal of Statistical Software 25: 1-18. [CrossRef]

Lew-Williams, Casey, and Anne Fernald. 2010. Real-time processing of gender-marked articles by native and non-native Spanish speakers. Journal of Memory and Language 63: 447-64. [CrossRef]

Libben, Maya R., and Debra A. Titone. 2009. Bilingual lexical access in context: Evidence from eye movements during reading. Journal of Experimental Psychology: Learning, Memory, and Cognition 35: 381-90. [CrossRef]

Long, Jacob A. 2019. Interactions: Comprehensive, User-Friendly Toolkit for Probing Interactions. R package Version 1.1.0. Available online: https: / / cran.r-project.org/package=interactions (accessed on 20 June 2021).

Lopukhina, Anastasiya, Konstantin Lopukhin, and Anna Laurinavichyute. 2021. Morphosyntactic but not lexical corpus-based probabilities can substitute for cloze probabilities in reading experiments. PLoS ONE 16: e0246133. [CrossRef]

Luchkina, Tatiana, Tania Ionin, Natalia Lysenko, Anastasia Stoops, and Nadezhda Suvorkina. 2021. Evaluating the Russian language proficiency of bilingual and Second Language Learners of Russian. Languages 6: 83. [CrossRef]

Lüdecke, Daniel. 2017. sjPlot: Data Visualization for Statistics in Social Science (R Package Version 2.3.3). Available online: https: / /CRAN.Rproject.org / package $=s j P l o t$ (accessed on 20 June 2021).

Luke, Steven G., and Kiel Christianson. 2016. Limits on lexical prediction during reading. Cognitive Psychology 88: 22-60. [CrossRef] [PubMed]

Lyashevskaya, O. N., and S. A. Sharov. 2009. Chastotnyj Slovar'_Sovremennogo Russkogo Jazyka (na Materialakh Natsional'nogo_Korpusa Russkogo Jazyka) [Frequency Dictionary of Modern_Russian (Based on the Materials of the Russian National Corpus)]. Moscow: Azbukovnik.

Martin, Clara D., Guillaume Thierry, Jan-Rouke Kuipers, Bastien Boutonnet, Alice Foucart, and Albert Costa. 2013. Bilinguals reading in their second language do not predict upcoming words as native readers do. Journal of Memory and Language 69: 574-88. [CrossRef]

Mitsugi, Sanako. 2020. Generating predictions based on semantic categories in a second language: A case of numeral classifiers in Japanese. International Review of Applied Linguistics in Language Teaching 58: 323-49. [CrossRef]

Mitsugi, Sanako, and Brian Macwhinney. 2016. The use of case marking for predictive processing in second language Japanese. Bilingualism: Language and Cognition 19: 19-35. [CrossRef]

Molinaro, Nicola, Francesco Giannelli, Sendy Caffarra, and Clara Martin. 2017. Hierarchical levels of representation in language prediction: The influence of first language acquisition in highly proficient bilinguals. Cognition 164: 61-73. [CrossRef]

Montrul, Silvina A. 2008. Incomplete Acquisition in Bilingualism: Re-Examining the Age Factor. Amsterdam: John Benjamins Publishing Company, vol. 39. [CrossRef] 
Mor, Billy, and Anat Prior. 2021. Frequency and predictability effects in first and second language of different script bilinguals. Journal of Experimental Psychology: Learning, Memory, and Cognition. Advance online publication. [CrossRef]

Morales, Luis, Daniela Paolieri, Paola E. Dussias, Jorge R. Valdés Kroff, Chip Gerfen, and Maria T. Bajo. 2016. The gender congruency effect during bilingual spoken-word recognition. Bilingualism: Language and Cognition 19: 294-310. [CrossRef]

O'Regan, J. Kevin, and Arthur M. Jacobs. 1992. Optimal viewing position effect in word recognition: A challenge to current theory. Journal of Experimental Psychology: Human Perception and Performance 18: 185-97. [CrossRef]

Parshina, Olga, Anna K. Laurinavichyute, and Irina A. Sekerina. 2021a. Eye-movement benchmarks in Heritage Language reading. Bilingualism: Language and Cognition 24: 69-82. [CrossRef]

Parshina, Olga, Irina A. Sekerina, Anastasiya Lopukhina, and Titus von der Malsburg. 2021b. Monolingual and bilingual reading processes in Russian: An exploratory scanpath analysis. Reading Research Quarterly. Advance online publication. [CrossRef]

Pickering, Martin J., and Chiara Gambi. 2018. Predicting while comprehending language: A theory and review. Psychological Bulletin 144: 1002-44. [CrossRef] [PubMed]

Polinsky, Maria. 2006. Incomplete Acquisition: American Russian. Journal of Slavic Linguistics 14: 191-262.

Polinsky, Maria. 2018. Heritage Languages and Their Speakers, 1st ed. Cambridge: Cambridge University Press. [CrossRef]

Polinsky, Maria, and Gregory Scontras. 2020. Understanding heritage languages. Bilingualism: Language and Cognition 23: 4-20. [CrossRef]

R Core Team. 2021. R: A Language and Environment for Statistical Computing. Vienna: R Foundation for Statistical Computing, Available online: https:/ / www.R-project.org/ (accessed on 20 June 2021).

Rayner, Keith. 2009. The 35th Sir Frederick Bartlett Lecture: Eye movements and attention in reading, scene perception, and visual search. Quarterly Journal of Experimental Psychology 62: 1457-506. [CrossRef] [PubMed]

Schlenter, Judith, and Claudia Felser. 2021. Second language prediction ability across different linguistic domains. In Prediction in Second-Language Processing and Learning. Edited by E. Kaan and T. Grüter. Amsterdam: John Benjamins Publishing Company, pp. 48-68. [CrossRef]

Scontras, Gregory, Zuzanna Fuchs, and Maria Polinsky. 2015. Heritage language and linguistic theory. Frontiers in Psychology 6: 1545. [CrossRef]

Sekerina, Irina A. 2015. Predictions, fast and slow. Linguistic Approaches to Bilingualism 5: 532-36. [CrossRef]

Staub, Adrian, and Keith Rayner. 2007. Eye movements and on-line comprehension processes. In The Oxford Handbook of Psycholinguistics. Edited by M. G. Gaskell. Oxford: Oxford University Press, pp. 325-42.

Taylor, Wilson L. 1953. “Cloze Procedure": A New Tool for Measuring Readability. Journalism Quarterly 30: 415-33. [CrossRef]

van Bergen, Geertje, and Monique Flecken. 2017. Putting things in new places: Linguistic experience modulates the predictive power of placement verb semantics. Journal of Memory and Language 92: 26-42. [CrossRef]

Whitford, Veronica, and Debra Titone. 2017. The effects of word frequency and word predictability during first- and second-language paragraph reading in bilingual older and younger adults. Psychology and Aging 32: 158-77. [CrossRef]

Woodcock, Richard W. 2011. Wookcock Reading Mastery Tests Third Edition Manual. Oxford: Pearson. 